\title{
Resonant growth in simple systems
}

\section{Gordon, R A}

\section{Published in:}

American Journal of Physics

Link to article, DOI:

10.1119/1.13430

Publication date:

1983

\section{Document Version}

Publisher's PDF, also known as Version of record

Link back to DTU Orbit

\section{Citation (APA):}

Gordon, R. A. (1983). Resonant growth in simple systems. American Journal of Physics, 51(1), $22-24$. https://doi.org/10.1119/1.13430

\section{General rights}

Copyright and moral rights for the publications made accessible in the public portal are retained by the authors and/or other copyright owners and it is a condition of accessing publications that users recognise and abide by the legal requirements associated with these rights.

- Users may download and print one copy of any publication from the public portal for the purpose of private study or research.

- You may not further distribute the material or use it for any profit-making activity or commercial gain

- You may freely distribute the URL identifying the publication in the public portal

If you believe that this document breaches copyright please contact us providing details, and we will remove access to the work immediately and investigate your claim 
${ }^{5}$ D. P. Marsh, Am. J. Phys. 48, 933 (1980).

${ }^{6} \mathrm{Marvin} \mathrm{L}$. De Jong, Programming and Interfacing the 6502 (Sams, Indianapolis, IN, 1980), pp. 218-235, 326-359.

${ }^{7}$ Listings and a cassette tape containing the computer recordings of these and other programs may be obtained by sending a blank cassette tape to the authors.

${ }^{8}$ Central Scientific Company, Cenco Selective Experiments in Physics (Central Scientific, Chicago, 1981-82), p. 6.

${ }^{9}$ F. W. Sears, M. W. Zemansky, and H. D. Young, University Physics, 6th ed. (Addison-Wesley, Reading, MA, 1982), p. 237.

\title{
Resonant growth in simple systems
}

\author{
R. A. Gordon \\ Physics Laboratory I, The Technical University of Denmark, DK-2800 Lyngby, Denmark
}

(Received 7 April 1980; accepted for publication 24 March 1982)

\begin{abstract}
It is shown that a simple extension of the usual sinusoidal trial solution in elementary textbooks to include a time-dependent amplitude of oscillation-a special case of the general KryloffBogoliuboff solution-makes it possible to give a simple unified treatment of resonant growth in simple systems at a level readily accessible to beginning physics students.
\end{abstract}

\section{INTRODUCTION}

The excitation of resonance in simple sytems is generally presented in elementary textbooks as a steady-state phenomenon in which a particular linear form of damping is assumed. ${ }^{1-3}$ Such an approach, however, tends to obscure the manner in which the resonance growth always takes place in simple systems and is, of course, completely unable to describe important resonance phenomena for which linear damping is inappropriate or where simple steady-state solutions do not exist. Thus, for example, the linear increase in the amplitude of oscillation in time for the usual case of resonance as well as a number of important resonance phenomena such as parametric resonance are generally ignored or treated in a very sketchy fashion in elementary textbooks. It is the purpose of this brief article to describe a simple extension of the trial solution conventionally used in elementary textbooks which makes it possible to give a unified description of various resonance phenomena in a form which is readily accessible to beginning physics students.

\section{TRIAL SOLUTION}

The equation of motion for a simple harmonic oscillator in the presence of a sinusoidal perturbation $f_{\text {pert }}(t)$ of amplitude $f(t)$ can be written as ${ }^{4}$

$$
\ddot{x}+\omega_{0}^{2} x=f_{\text {pert }}(t)=f(t) \cos (\omega t+\phi),
$$

where $x$ is the displacement of the oscillator from its unperturbed equilibrium position, $x=0, \omega_{0}$ is the angular frequency in the absence of the sinusoidal perturbation, and $\omega$ and $\phi$, respectively, are the angular frequency and phase of the perturbation, taken to be constants. It is well known ${ }^{5}$ that the general solution $x_{\text {gen }}(t)$ of Eq. (1) can be written as

$$
x_{\text {gen }}(t)=x_{\text {gen }}\left(t, C_{1}, C_{2}\right) \text {, }
$$

where $C_{1}$ and $C_{2}$ are arbitrary constants determined by the initial conditions, and where $x_{\text {gen }}(t)$ approaches the solution $x_{h}(t)$ of the homogeneous, unperturbed, equation of motion $[f f(t) \mid=0$ in Eq. (1)] in the limit of vanishingly small perturbations, $|f(t)| \rightarrow 0$, i.e.,

$$
\begin{aligned}
& x_{\text {gen }}\left(t, C_{1}, C_{2}\right) \rightarrow x_{h}(t) \\
& \quad=A \cos \omega_{0} t+B \sin \omega_{0} t \quad \text { as }|f(t)| \rightarrow 0,
\end{aligned}
$$

where $A$ and $B$ are constants.

In general, $x_{\text {gen }}\left(t, C_{1}, C_{2}\right)$ is quite complex and in analogy with the approach used in elementary textbooks, we restrict our attention to cases for which the general solution of Eq. (1) can be expressed in terms of a simple sinusoidal trial solution ${ }^{6} x_{p}(t)$ at a constant angular frequency $\Omega$, differing in phase by a constant amount, $\phi$, from that of the sinusoidal perturbation but with an amplitude $X(t)$, which is a function of time $e^{7,8}$.

$$
x_{p}(t)=X \cos \Omega t \quad X=X(t) .
$$

The values of $\Omega, \phi$, and $X(t)$ are then chosen so that Eq. (4) is a solution (or a good approximate solution) of Eq. (1). The general solution of Eq. (1) is then found by adding the appropriate solution of the homogeneous equation of motion such that Eqs. (2) and (3) are satisfied. A solution of this form differs from that given in standard elementary textbooks only in assuming that the amplitude of oscillation can be a function of time but has the advantage that both constant and time-dependent amplitudes can be described using the same formulation. At the same time, Eq. (4) is much simpler to apply than the completely general, exact Kryloff-Bogoliuboff solution ${ }^{5,10}$ of Eq. (1) which differs from Eq. (4) in taking the angular frequency $\Omega$, and the phase difference $\phi$ to be functions of time. ${ }^{11}$ We now illustrate how Eq. (4) can be used to find a general solution of Eq. (1) which is exact for the standard form of resonance.

\section{STANDARD RESONANCE}

In this case, the amplitude of the sinusoidal perturbation, $f(t)$, is constant and the equation of motion, Eq. (1), can thus be written as

$$
\ddot{x}+\omega_{0}^{2} x=f_{\text {pert }}(t)=f \cos (\omega t+\phi) \quad f=\text { const. }
$$

For such a perturbation, the general solution, as is well 
known, will be equal to the sum of any particular solution of Eq. (5) plus the solution of the homogeneous equation of motion [viz. Eq. (3)]. Thus the trial solution given above [Eq. (4)] represents, in generalized form, the usual particular solution given in elementary textbooks, plus one or both of the two linearly independent solutions $\left(\cos \omega_{0} t\right.$ and $\left.\sin \omega_{0} t\right)$ of the homogeneous equation of motion [viz. Eq. (3)].

An exact solution of Eq. (5), valid for the case of a time dependent amplitude of oscillation $X(t)$, can be found for $\Omega=\omega$ in Eq. (4), i.e., for a sinusoidal oscillation at the same angular frequency as that of the perturbation. To see this we take ${ }^{12}$

$$
\Omega=\omega
$$

and substitute Eq. (4) into Eq. (5) yielding

$$
\begin{aligned}
& {\left[\ddot{X}+\left(\omega_{0}^{2}-\omega^{2}\right) X\right] \cos \omega t-[2 \omega \dot{X}] \sin \omega t} \\
& \quad=f \cos (\omega t+\phi) .
\end{aligned}
$$

Equation (7) will be satisfied for all values of $t$ if the values of $\phi$ and $X(t)$ are such that the coefficients of the sin $\omega t$ and $\cos \omega t$ terms on both sides of Eq. (7) are equal. This leads to two simultaneous equations:

$$
\begin{aligned}
& \ddot{X}+\left(\omega_{0}^{2}-\omega^{2}\right) X=f \cos \phi, \\
& 2 \omega \dot{X}=f \sin \phi .
\end{aligned}
$$

Equation (9) implies that Eq. (8) can be rewritten as

$$
\left(\omega_{0}^{2}-\omega^{2}\right) X=f \cos \phi
$$

Equations (9) and (10) will be satisfied simultaneously only for special combinations of $\omega$ and $\phi$. Thus for $\omega \neq \omega_{0}$, Eq. (10) implies that a constant amplitude of oscillation $X$ is possible, i.e.,

$$
X=(f \cos \phi) /\left(\omega_{0}^{2}-\omega^{2}\right) \quad \omega \neq \omega_{0} .
$$

On the other hand, such a constant amplitude for $X$ will contradict Eq. (9) unless ${ }^{9}$

$$
\sin \phi=0 \quad \phi=0, \pi \text {. }
$$

It can readily be seen by inspection of Eqs. (4), (5), and (11), or by a simple displacement of $(-\phi / \omega)$ in the time origin, ${ }^{8}$ that the two values of $\phi(0$ or $\pi)$ represent the same trial solution corresponding to an oscillation of amplitude $f /\left(\omega_{0}^{2}-\omega^{2}\right)$ which is in phase with the sinusoidal perturbation for $\omega<\omega_{0}$ and $180^{\circ}$ out of phase for $\omega>\omega_{0}$. Thus without any loss of generality, $\phi$ can be taken equal to 0 . Adding the solution of the homogeneous equation of motion to the trial solution then yields the general solution, $x_{\text {gen }}(t)$ for $\omega \neq \omega_{0}$ which satisfies Eqs. (2) and (3) above:

$x_{\text {gen }}(t)=(f \cos \omega t) /\left(\omega_{0}^{2}-\omega^{2}\right)+A \cos \omega_{0} t+B \sin \omega_{0} t$,

$f_{\text {pert }}(t)=f \cos \omega t \quad \omega \neq \omega_{0}$.

This corresponds to the usual constant-amplitude, steadystate solution given in elementary textbooks.

In the remaining resonance case, $\omega=\omega_{0}$, Eq. (10) implies that

$$
\cos \phi=0 \quad \phi=\pi / 2,3 \pi / 2 \text {, }
$$

leading to a nonzero solution for $\dot{X}$ in Eq. (9):

$$
X=(f t \sin \phi) /\left(2 \omega_{0}\right)+C \quad \omega=\omega_{0}
$$

where $C$ is an arbitrary constant. Again it can be readily seen by inspection of Eqs. (4), (5), and (15), or by a simple displacement of $(-\phi / \omega)$ in the time origin, ${ }^{8}$ that (apart from an arbitrary multiple of one of the constant amplitude solutions of the homogeneous solution) the two values of $\phi$

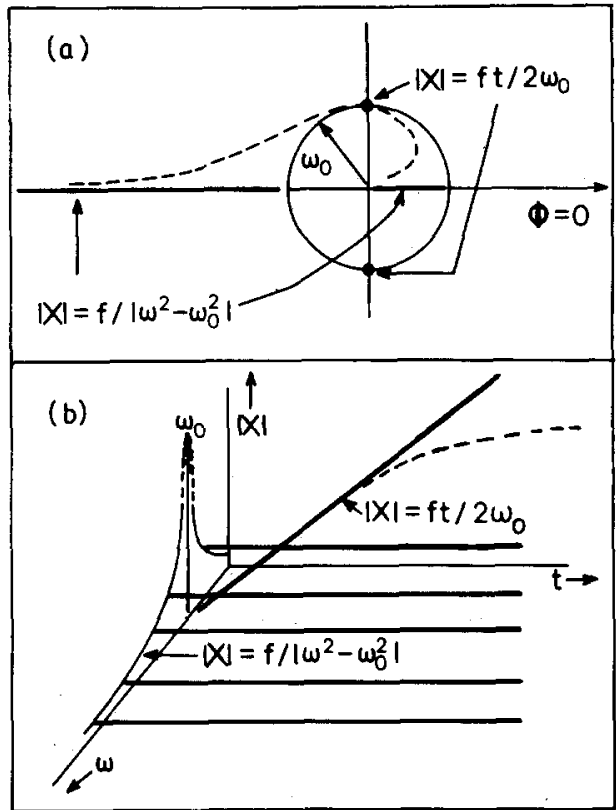

Fig. 1. Standard form of resonance. (a) Polar plot of the angular frequency $\omega$ of the sinusoidal perturbation (heavy lines and points) as a function of the phase difference $\phi$ (measured clockwise from the $\phi=0$ reference direction) between the sinusoidal perturbation and the constant amplitude and resonance trial solutions [Eqs. (12) and (14), respectively] of the equation of motion, Eq. (5). The absolute value of the amplitude of oscillation $|X|$ of the constant amplitude [Eq. (11)] and resonance [Eq. (15)] trial solutions of Eq. (5), associated with different values of $\omega$, as a function of the time $t$ are indicated by arrows. The arbitrary additive constant in the resonance trial solution [Eq. (15)] has been taken equal to zero for convenience. (b) $|X|$ (heavy lines) as a function of $\omega$ and $t$ for constant amplitude and resonance trial solutions. The dotted lines qualitatively depict the $\omega$ vs $\phi$ relationship [Fig. 1(a)] as well as $|X|$ as a function of $\omega$ and $t$ in the case of linear damping, where the resonance response will be broadened in angular frequency, the amplitude of oscillation limited for larger values of time, and the discontinuity in the $\omega$ vs $\phi$ curve removed.

in Eq. (14) $(\pi / 2$ and $3 \pi / 2)$ represent the same trial solution corresponding to an oscillation of amplitude $(f t) /\left(2 \omega_{0}\right)$ which lies $90^{\circ}$ in phase behind the sinusoidal perturbation. Thus without any loss of generality, only one of the two values of $\phi$ need be considered. Displacing the time origin by an amount $(-\phi / \omega)^{8}$ for ease of comparison with the steady-state solution [Eq. (13)], and adding the solution of the homogeneous equation then yields the general solution for $\omega=\omega_{0}$ which satisfies Eqs. (2) and (3):

$x_{\text {gen }}(t)=\left(f t \sin \omega_{0} t\right) /\left(2 \omega_{0}\right)+A^{\prime} \cos \omega_{0} t+B^{\prime} \sin \omega_{0} t$,

$f_{\text {pert }}(t)=f \cos \omega t \quad \omega=\omega_{0}$,

where $A^{\prime}$ and $B^{\prime}$ are arbitrary constants. It is worth noting that the monotonic linear increase in time of the oscillation amplitude $90^{\circ}$ in phase behind the sinusoidal perturbation at resonance $\omega=\omega_{0}$, can not be removed by any choice of the constants $A^{\prime}$ or $B^{\prime}$ and is hence independent of the choice of initial conditions, i.e., the values of $x_{\text {gen }}(t=0)$, $\dot{x}_{\text {gen }}(t=0)$. The various relations between the amplitude frequency, and phase for both constant amplitude and resonance trial solutions are summarized graphically in Fig. 1.

\section{DISCUSSION}

It is worth emphasizing that the method described in detail here can also be readily applied to find sinusoidal 
solutions with a time-varying amplitude of oscillation for equations of motion much more complicated than Eq. (1). Thus, for example, the inclusion of a linear damping term can be readily shown to lead to a saturation in the linear increase of oscillation amplitude in time while removing the discontinuity in the frequency-phase difference dependence as indicated in Fig. 1. In similar fashion it can also be shown that a single sinusoidal trial solution provides an excellent approximate solution in the case of parametric resonance for sufficiently small sinusoidal perturbations of one of the parameters determining the unperturbed oscillation frequency $\omega_{0}$ of the system in question. ${ }^{13}$

${ }^{1}$ G. R. Fowler, Analytical Mechanics (Holt-Saunders, London, 1970).

${ }^{2}$ D. Kleppner and R. J. Kolenkow, An Introduction to Mechanics (McGraw-Hill, Düsseldorf, 1973).

${ }^{3}$ R. P. Feynman, R. B. Leighton, and M. Sands, The Feynman Lectures on Physics, Vol. I (Addison-Wesley, Amsterdam, 1975).

${ }^{4}$ The $\operatorname{dot}(\cdot)$ denotes differention with respect to time $t$, i.e., $(\cdot) \equiv(d / d t)$.

${ }^{5}$ G. A. Korn and T. M. Korn, Mathematical Handbook (McGraw-Hill, London, 1961).

${ }^{6}$ Here $x_{p}(t)$ is referred to as a trial solution to distinguish it from the more restrictive term, particular solution, to which it is equivalent only in special cases, e.g., the case of standard resonance as discussed below.

'In general, Eq. (4) can be expected to provide a good description of resonant growth in simple systems whenever the phase difference $\phi$ can be regarded as a sufficiently slowly varying function of time that $\dot{\phi} \cong 0$. It is worth noting that the method used here has also been applied to find the solution of the homogeneous equation although it has not, to the authors knowledge, been used to treat the resonance phenomena discussed here in standaard elementary textbooks; H. Greeburg, Am. J. Phys. 44, 1135 (1976).

${ }^{8}$ Solutions of Eq. (1) for different values of the phase difference, $\phi$, can be compared for the same perturbation $f_{\text {pert }}(t)$ by a simple displacement of the time origin by an amount $-\phi / \omega$ in the trial solution [Eq. (4)]. Equivalent values of the phase difference $\phi$ could, of course, also be found by taking the sinusoidal perturbation to be $f(t) \cos \omega t$ with the trial solution given by $X(t) \cos (\Omega t+\phi)$ although there is no advantage to such an approach since the algebra is significantly more tedious and is not necessary to demonstrate the relation between the phase difference and the angular frequency as well as the growth in oscillation amplitude at resonance.

${ }^{9}$ The value of the phase difference $\phi$ is taken to lie between 0 and $2 \pi$, $0 \leqslant \phi<2 \pi$.

${ }^{10} \mathrm{~N}$. Minorsky, Nonlinear Oscillations (Krieger, New York, 1972).

"It is worthwhile pointing out that the trial solution used here also differs from the so-called variation of parameters method in which the angular frequency $\Omega$ is taken to be equal to the unperturbed angular frequency $\omega_{0}$ while the phase difference $\phi$ is time dependent. The variation of parameters method will lead to the same result as that given in the text for the case of standard resonance but is not readily applicable to the case of parametric resonance. L. A. Pipes and L. R. Harvill, Applied Mathematics for Engineers and Scientists (McGraw-Hill, Düsseldorf, 1970).

${ }^{12}$ The choice of $\Omega=\omega$ can also be motivated by requiring that Eq. (4) be valid solution of Eq. (1) for all values of time with the additional requirement that $X(t)$ be a slowly varying function of time in comparison with both periods of oscillation $2 \pi / \omega$ and $2 \pi / \Omega$. In similar fashion the assumption that the amplitude of oscillation be as simple as possible (e.g., $\dot{X}=0$ or $\ddot{X}=0$ ) would also lead to the condition $\Omega=\omega$.

${ }^{13}$ L. D. Landau and E. M. Lifshitz, Mechanics (Pergamon, Paris, 1969); Report No. 235, Physics Lab. I, Technical University of Denmark, DK-2800 Lyngby, Denmark.

\title{
Petrov classification: An elementary approach
}

\author{
T. M. Kalotas \\ Physics Department, La Trobe University, Bundoora, 3083, Australia \\ C. J. Eliezer \\ Department of Applied Mathematics, La Trobe University, Bundoora, 3083, Australia
}

(Received 23 July 1981; accepted for publication 19 February 1982)

We reduce the length of the usual algebraic classification scheme of the Weyl tensor by avoiding the step centered around its reduction to canonical form. Instead the different algebraic types are established more economically via the elementary approach of constructing explicit examples.

\section{INTRODUCTION}

The classification of Einstein's gravitational field into a finite number of distinct algebraic types was first carried out by Petrov, ${ }^{1}$ and has played an important part in the development and understanding of general relativity. In particular it has facilitated the study and generation of exact solutions of the Einstein field equations ${ }^{2,3}$ and in some instances has allowed one to draw certain general conclusions about the physical nature of a solution belonging to a particular Petrov type, a case in point being the gravitational radiating solutions.
Although the subject of algebraic classification in one or more of its alternative expositions ${ }^{4-8}$ is by now familiar to those researching in relativity, the topic is still generally considered to be too advanced for inclusion in an undergraduate curriculum. This is unfortunate since the basic idea of classifying a matrix via its eigenvalues and eigenvectors, is already familiar to those students who are at the stage of learning about general relativity and so the essentials of algebraic classification should certainly not be too hard for them. To make the subject more palatable it then appears that a reduction in length is required more than anything else and this is where the approach which we advocate in 